\title{
The importance of physical structure in solar dynamo models
}

\author{
A. Phillips ${ }^{1, \star}$, J. Brooke $e^{1,2}$, and D. Moss ${ }^{1}$ \\ 1 Department of Mathematics, University of Manchester, Manchester M13 9PL, UK \\ 2 Manchester Computing, University of Manchester, Manchester M13 9PL, UK
}

Received 12 February 2002 / Accepted 11 June 2002

\begin{abstract}
We investigate numerically a simplified, interface-type dynamo model with two spatial dimensions, using Cartesian geometry, by examining the effect of various plausible modifications in the physics and geometry in order to approximate conditions in the solar convection zone and overshoot layer. We describe such modifications as changes to the structure of the model. We are particularly interested in the effects of such structural changes on models which incorporate the nonlinear feedback between the magnetic field and the rotational profile via the Malkus-Proctor feedback mechanism, since this form of nonlinearity can reproduce important phenomena such as torsional oscillations and grand minima. We also examine recent claims that, with such a nonlinearity, there exists generic behaviour which is robust to changes in the structure of the model. We find that there is no generic sequence of behaviour for these interface models, but rather that altering physical details of the model can produce major changes in observable signatures, from the very start of the bifurcation sequences. A significant overall result is that the configuration of the migrating field belts can be strongly dependent on depth. Thus, the question of the exact regions in which solar activity tracers (e.g. sunspots) are formed becomes important for comparison of dynamo theory with solar observations.
\end{abstract}

Key words. magnetic fields - MHD - Sun: magnetic fields

\section{Introduction}

Observations of solar activity tracers provide a number of challenging issues for dynamo theory. For example, numerical simulations of buoyantly rising magnetic flux tubes suggest that toroidal field strengths at the base of the solar convection zone are of the order of $10^{4}-10^{5} \mathrm{G}$ (Fan et al. 1993; Caligari et al. 1995, 1998). These estimates pose two major problems for solar dynamo theorists, the first being that magnetic flux tubes with initial field strengths greater than $O\left(10^{2}\right) \mathrm{G}$ are predicted to rise to the solar surface on timescales much shorter than the 22 year cycle period (Parker 1975; Moreno-Insertis 1983). The second difficulty is that the theoretically predicted field strengths are at least one order of magnitude greater than the equipartition value $B_{\text {eq }} \sim 3 \mathrm{kG}$.

Parker (1993) suggested that these difficulties might be avoided in a model in which the $\alpha$-effect and $\omega$-effect (differential rotation) are confined to two separate layers. This interface model allows strong toroidal fields to be generated and "stored" beneath the convection zone for lengths of time comparable with the 22 year cycle period, whilst weaker poloidal fields are produced in the convection zone by the action of cyclonic

Send offprint requests to: D. Moss, e-mail: moss@ma .man . ac . uk

* Present address: NASA Goddard Space Flight Center, Code 931, Greenbelt, MD 20771, USA. turbulence. More recent studies of interface-type dynamo models (Charbonneau \& MacGregor 1997; Markiel \& Thomas 1999) have shown that by restricting the $\alpha$-effect to regions where the magnetic field is weak, difficulties associated with $\alpha$ quenching may be avoided, leading to magnetic field strengths more reconcilable with observations.

The interface dynamo has provided the basis for a class of models which utilise large scale feedback of the magnetic field on the rotational profile, and are capable of reproducing solutions which capture some of the essential features of solar magnetic activity (Tobias 1996, 1997, hereafter T96, T97). Interestingly, such features are also seen in very simplified systems of ordinary differential equations (ODEs) designed to reproduce the symmetries of the solar dynamo (Tobias et al. 1995; Knobloch \& Landsberg 1996). It can be shown that the behaviour of these low-order systems and the numerical models based on the solution of partial differential equations (PDEs) are similar in important qualitative aspects (Knobloch et al. 1998). In the solar context, it is of special importance that such models provide a possible explanation for episodes of grand minima in magnetic activity, and for symmetry changes that are believed to have occured at the time of the Maunder minimum, when observed sunspots were largely restricted to the southern hemisphere (Ribes \& Nesme-Ribes 1993). This led Knobloch et al. to postulate that such behaviour is generic, since the 
results of the low order models are generic within the framework of normal form theory, and are therefore likely to be observed in a wide range of stellar dynamo models. Hence they have a good probability of being observed in the magnetic activity of solar-type stars.

However, the concept of genericity in dynamical systems does not directly support such a conclusion. One definition of generic behaviour is that it is observed in a set of non-zero measure in the phase space of all solutions, but it may still only occupy a "small volume" of this phase space and thus may be observed only rarely. Also, normal form theory in ODEs is critically dependent on the structure of the bifurcation itself, and the connection between the symmetries of the normal form system and the physical symmetries of the system of PDEs that the low order system is attempting to model is not in any sense direct or unambiguous. Dynamo models have also been shown to exhibit structural fragility, in that small changes in model dependent features, such as boundary conditions, can lead to qualitative changes in behaviour (Tavakol et al. 1995). Instead of investigating genericity, we prefer to use the more physically motivated concept of stuctural robustness. We try to identify which features of the solutions persist when certain changes are made to the basic model. Such changes, e.g. in the boundary conditions or the aspect ratio of the two regions of the interface, are described as structural because they leave key features of the physics of the interface model unaltered. Examples of changes which we would regard going outside the structure of the model would be the abandonment of the interface concept, or a radical change in the nonlinearity, e.g. to flux loss via buoyancy or to an alpha-quenching mechanism, rather than the dynamical Malkus-Proctor feedback on the velocity field. Our structural changes are more to do with details of the model, and they are difficult to map on to low-order idealisations, or truncations. The questions of genericity and structural robustness in dynamical systems raise important issues which we cannot fully address here; see, for example, Guckenheimer \& Holmes (1983, Ch. 5) for a fuller discussion.

Another important idealisation of the Parker interface dynamo that needs to be investigated is its assumption of a discontinuity in model coefficients, such as magnetic diffusivity. This unphysical aspect needs to be explored in numerical models that attempt to capture a transition between the convective and overshoot regions. In this paper, we investigate the effect of several modifications of the original Parker-type model which attempt to introduce in a controlled fashion various features of the solar convective and overshoot regions, as revealed by helioseismological observations, in order to estimate the sensitivity of the bifurcation sequence to such changes. Our reference point is the numerical model of the Parker dynamo described in T96 and T97, since the bifurcation sequence in this model is very thoroughly analyzed, and related to the results of loworder models where more rigorous statements about the form of the bifurcations can be investigated.

This paper is structured as follows. We derive a set of generalised MHD dynamo equations incorporating an idealised model for the large-scale feedback of the Lorentz force on a zero-order velocity profile in Sect. 2 . The behaviour of the basic reference configuration of this model (as in T96, T97) is summarized in Sect. 3, paying particular attention to the form of the bifurcation sequence. Several plausible modifications to the this model are analyzed in Sect. 4. This is followed in Sect. 5 by a discussion of the magnetic field structures and migration patterns for the various models, with reference to important known phenomena of the solar cycle. Finally, our overall conclusions are summarised in Sect. 6.

\section{Dynamo equations}

The standard mean-field induction equation (Moffatt 1978) is

$\frac{\partial \boldsymbol{B}}{\partial t}=\nabla \times[(\boldsymbol{V} \times \boldsymbol{B})+\alpha \boldsymbol{B}-\eta \nabla \times \boldsymbol{B}]$,

where $\boldsymbol{B}$ and $\boldsymbol{V}$ are the large-scale (mean) magnetic and velocity fields, $\alpha$ is the $\alpha$-effect coefficent, and $\eta$ is the (turbulent) magnetic diffusivity. Assuming axisymmetry and neglecting meridional circulation effects, the toroidal component of the momentum equation is then

$\rho \frac{\partial V_{\mathrm{T}}}{\partial t}=(\nabla \cdot \sigma)_{\mathrm{T}}+(\boldsymbol{j} \times \boldsymbol{B})_{\mathrm{T}}$

Here, $\rho$ is the plasma density, $\nabla \cdot \sigma$ is the stress term, and $j$ is the large-scale current density.

Equations (1) and (2) are solved in two spatial dimensions, with dynamo action confined to a rectangular box. In a Cartesian co-ordinate system $(x, y, z)$, the latitude is represented by the co-ordinate $0 \leq x \leq 2 L$, with the north (south) pole at $x=0(x=2 L)$ and the equator at $x=L$. The co-ordinate $z_{1} \leq z \leq l$ corresponds to the radial direction, with the overshoot layer $\left(z_{1} \leq z<0\right)$ and convection zone $(0<z \leq l)$ separated by a notional "interface" at $z=0$. The unit vector $\hat{x}$ points tangentially south along the meridian, $\hat{\boldsymbol{y}}$ in the direction of rotation, and $\hat{z}$ radially outwards. The $y$-direction corresponds to longitude, and following the assumption that our model is related to an axisymmetric spherical model, there is no $y$-dependence in our analysis.

As $\partial / \partial y \equiv 0$, the magnetic field may be written as $\boldsymbol{B}=$ $B \hat{\boldsymbol{y}}+\nabla \times(A \hat{\boldsymbol{y}})$. Here, $A(x, z, t)$ and $B(x, z, t)$ are scalar functions which correspond to the poloidal vector potential and toroidal field. Thus, $\nabla \cdot \boldsymbol{B}=0$ automatically. Assuming $\eta$ to be a function of $z$ alone, the velocity field may be expressed as $\boldsymbol{V}=V \hat{\boldsymbol{y}}-\nabla \eta / 2=(U+v) \hat{\boldsymbol{y}}-1 / 2(\partial \eta / \partial z) \hat{\boldsymbol{z}}$. The scalar function $V(x, z, t)$ has been split into two parts: $U(x, z)$ and $v(x, z, t)$. The first of these corresponds to the (time averaged) rotation law (as, for example, inferred from helioseismology), whereas the second represents deviations from the zero-order velocity state driven by Lorentz forces, which act to modify the differential rotation and to quench the $\omega$-effect. An additional term has been included to model the effects of turbulent diamagnetism (Zel'dovich 1956; Vainshtein \& Zel'dovich 1972; Roberts \& Soward 1975), which acts to transport magnetic flux from regions of high to low diffusivity.

In the $\alpha \omega$ limit, differential rotation is assumed to be solely responsible for the generation of toroidal field from poloidal. Selected computations using an $\alpha^{2} \omega$ model suggest this approximation is valid for all of the cases presented in this paper. From the definition of the model, it is clear that the system will 
return to the zero-order state in the absence of a magnetic field. Equating toroidal and poloidal components of Eq. (1) gives equations for the evolution of $A$ and $B$, whilst a similar expression for $\partial v / \partial t$ may be derived from Eq. (2). The resulting equations are then non-dimensionalised by setting

$x=l \tilde{x}, \quad z=l \tilde{z}, \quad t=l^{2} \tilde{t} / \eta_{0}, \quad A=A_{0} \tilde{A}$,

$B=B_{0} \tilde{B}, \quad U=l \Omega_{0} \tilde{U}, \quad v=l \Omega_{0} \tilde{v}, \quad \alpha=\alpha_{0} \tilde{\alpha}$,

$\eta=\eta_{0} \tilde{\eta}, \quad v=v_{0} \tilde{v}, \quad \rho=\rho_{0} \tilde{\rho}$,

where $l$ is the physical extent of the convection zone measured from the interface to the surface, $\alpha_{0}, \Omega_{0}, \eta_{0}, v_{0}$ and $\rho_{0}$ are typical values of the $\alpha$-effect, angular velocity, magnetic diffusivity, viscosity and density, and $l \Omega_{0}$ is the surface (solar) equatorial velocity.

Since the dynamo is of $\alpha \omega$ type, the field amplitude ratio $A_{0} / B_{0}$ may be chosen such that the combined strengths of the $\alpha$-effect and $\omega$-effect are governed by a single parameter. We therefore define $A_{0} / B_{0}=\alpha_{0} l^{2} / \eta_{0}$. Assuming the density to be constant and omitting the tildes, we obtain

$$
\begin{aligned}
\frac{\partial A}{\partial t}= & \eta \nabla^{2} A+\frac{1}{2} \frac{\partial A}{\partial z} \frac{\partial \eta}{\partial z}+\alpha B, \\
\frac{\partial B}{\partial t}= & \eta \nabla^{2} B+\frac{3}{2} \frac{\partial B}{\partial z} \frac{\partial \eta}{\partial z}+\frac{1}{2} B \frac{\partial^{2} \eta}{\partial z^{2}} \\
& +D\left[\frac{\partial A}{\partial x}\left(\frac{\partial U}{\partial z}+\frac{\partial v}{\partial z}\right)-\frac{\partial A}{\partial z}\left(\frac{\partial U}{\partial x}+\frac{\partial v}{\partial x}\right)\right],
\end{aligned}
$$

and

$\frac{\partial v}{\partial t}=\tau\left[\eta \nabla^{2} v+\frac{\partial \eta}{\partial z} \frac{\partial v}{\partial z}\right]+\operatorname{sgn}(D)\left[\frac{\partial A}{\partial x} \frac{\partial B}{\partial z}-\frac{\partial A}{\partial z} \frac{\partial B}{\partial x}\right]$.

Here, we have put $B_{0}^{2}=\rho_{0} \Omega_{0} \mu_{0} \eta_{0} /\left|R_{\alpha}\right|$, so that the nonlinear terms in Eq. (5) are multiplied by a coefficient of magnitude unity. The controlling parameters are

$D=\frac{\alpha_{0} \Omega_{0} l^{3}}{\eta_{0}^{2}}, \quad \tau=\frac{v_{0}}{\eta_{0}}$,

where $D$ is the dynamo number and $\tau$ is the magnetic Prandtl number. In the investigations which follow, we set $\tau=0.1$ to facilitate comparison with T97. The behaviour in the limit $\tau \rightarrow 0$ will be discussed in a future paper.

\section{The basic model}

The model described below is the same as that of T97. In contrast to the interface model of Parker (1993), the radial profiles of the $\alpha$-effect, $\omega$-effect and magnetic diffusivity are defined to be continuous. The $\alpha$-effect is given by

$\alpha=f(z) \cos \left(\frac{\pi x}{2 L}\right)$,

where

$f(z)= \begin{cases}0 & z \leq-0.75 \\ \frac{1}{2}[1+\tanh 5 z]\left[1-\mathrm{e}^{-25(z+0.75)^{2}}\right] & z>-0.75\end{cases}$

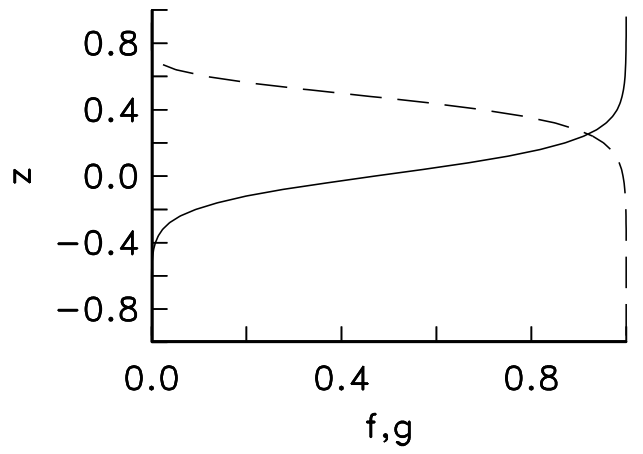

Fig. 1. Profiles of the functions $f(z)$ (solid) and $g(z)$ (dashed), which determine the radial dependence of the $\alpha$-effect and velocity shear respectively. The shear is concentrated in the overshoot layer $(z<0)$, whereas the $\alpha$-effect is strongest in the convection zone $(z>0)$. These profiles are similar to those presented in Fig. 1 of T97.

contains the $z$-dependence. The inclusion of the cosine term ensures that the $\alpha$-effect is antisymmetric about the equator.

Similarly, the zero-order velocity is defined as

$U(x, z)=u(z) \sin \left(\frac{\pi x}{2 L}\right)$.

Here, the angular velocity shear is assumed to be strictly radial, whilst a sine term has been included to mimic the effects of curvature, ensuring that $U=0$ at the poles (cf. Jennings \& Weiss 1991). The profile of the equatorial zero-order velocity $u(z)$ is determined from a prescribed shear function $g(z)=\mathrm{d} u / \mathrm{d} z$, given by

$g(z)= \begin{cases}\frac{1}{2}[1-\tanh 5(z-0.5)]\left[1-\mathrm{e}^{-25(z-0.75)^{2}}\right] & z<0.75 \\ 0 & z \geq 0.75\end{cases}$

Here, some of the parameters listed in T96 have been interchanged, to give a $g(z)$ profile in agreement with that presented in Fig. 1 of T97. (Our comparison with the T97 results, outlined below, confirms that this is appropriate.) Somewhat arbitrarily, the equatorial zero-order velocity at the surface is set to zero. Radial profiles of $f$ (solid) and $g$ (dashed) are shown in Fig. 1. Clearly, the velocity shear is concentrated in the overshoot layer, whilst the $\alpha$-effect is strongest in the convection zone. This is consistent with the quenching of cyclonic turbulence by strong toroidal fields. Note also the region of overlap $(0 \lesssim z \lesssim 0.5)$, where both the $\alpha$-effect and $\omega$-effect are at half maximum strength or greater. These profiles agree with those presented in Fig. 1 of T97. Here, we set $\eta(z)=1, z_{1}=-1$ and $L=4$.

Equations (3)-(5) are integrated subject to the boundary conditions

$A=B=v=0, \quad x=0,2 L$,
$A=B=v=0, \quad z=-1$,
$\frac{\partial A}{\partial z}=B=\frac{\partial v}{\partial z}=0, \quad z=1$,

so that the symmetry properties of the solution are not fixed. The constraints imposed on $A, B$ and $v$ at the poles follow from 
regularity of the magnetic and velocity fields. The physics at the upper and lower boundaries are less certain. At $z=-1$, the boundary conditions imply the inner core rotates rigidly, with no magnetic field generated beneath the overshoot layer. The constraints on the magnetic field at the surface are a local approximation to vacuum boundary conditions in Cartesian geometry, whilst the upper constraint on $v$ gives zero stress.

Since $\partial \Omega / \partial z>0$, pragmatically we restrict our attention to values of $D<0$ in order to achieve the correct (i.e. equatorward) sense of toroidal field migration (Parker 1979, 1993), without discussing the physics that would give $\alpha<0$ in the northern hemisphere. The MHD dynamo equations were integrated using a second-order Runge-Kutta scheme, with a time step of $\Delta t=10^{-4}$. Although computations were generally performed with 49 grid points in both the $x$ and $z$ directions, obtaining reliable results in the regime of high supercriticality required a spatial resolution of $99 \times 99$. Setting $v=\partial v / \partial t=0$ and integrating the linear problem (Eqs. (3) and (4)) gave critical dipolar and quadrupolar dynamo numbers of $D_{\mathrm{c}}(D) \approx-264$ and $D_{\mathrm{c}}(Q) \approx-310$, within $5 \%$ of the values of $D_{\mathrm{c}}(D) \approx-279$ and $D_{\mathrm{c}}(Q) \approx-325$ given in T97. The discrepancies may be ascribed to differences between the two solution methods.

To monitor our solutions, we define the three quantities

$E_{\mathrm{m}}=\frac{1}{V} \iint_{V} B^{2} \mathrm{~d} V, \quad E_{\mathrm{k}}=\frac{1}{V} \iint_{V} v^{2} \mathrm{~d} V$

$P=\frac{E_{\mathrm{q}}-E_{\mathrm{d}}}{E_{\mathrm{q}}+E_{\mathrm{d}}}$

Here, $E_{\mathrm{m}}$ and $E_{\mathrm{k}}$ are the energies associated with the toroidal magnetic field and the kinetic energy of the velocity perturbations, averaged over the spatial grid (the domain $V$ ). $E_{\mathrm{q}}$ and $E_{\mathrm{d}}$ are the energies associated with the symmetric and antisymmetric parts of the toroidal field, and $P$ is the field parity. For a purely quadrupolar/dipolar field, $P= \pm 1$. In addition, theoretical butterfly diagrams may be obtained by plotting "slices" of $B(x, t)$ at a given depth. For all such diagrams presented in this paper, positive values are represented by solid contours, negative fields by dotted contours, and zero fields by dashed contours.

To investigate the existence of multiple solutions, computations were performed for initial fields with parities $P(0) \approx 0$, $P(0)=-1$, and $P(0)=+1$, and dynamo numbers ranging from marginally supercritical to those in the (apparently) moderately chaotic regime. If two or more solution states were found to coexist over a given range of $D$, their stability was tested by introducing a perturbation of order unity to the toroidal field $B(x, z)$ of typical solutions at a centrally located grid point. For all of the cases discussed in this paper, this had no discernible longterm effect on the time series, indicating a large basin of stability. Although more than three stable solution branches may plausibly co-exist in some parameter regimes, investigating the behaviour for other values of $P(0)$ would be time consuming and impractical.

Figure 2 shows two bifurcation diagrams. The first of these corresponds to the results of T97. Here, estimates of $\left\langle E_{\mathrm{m}}\right\rangle$ (where the angled brackets denote the temporal average) were made from the time series presented in Figs. 2, 4, 6-9, 11

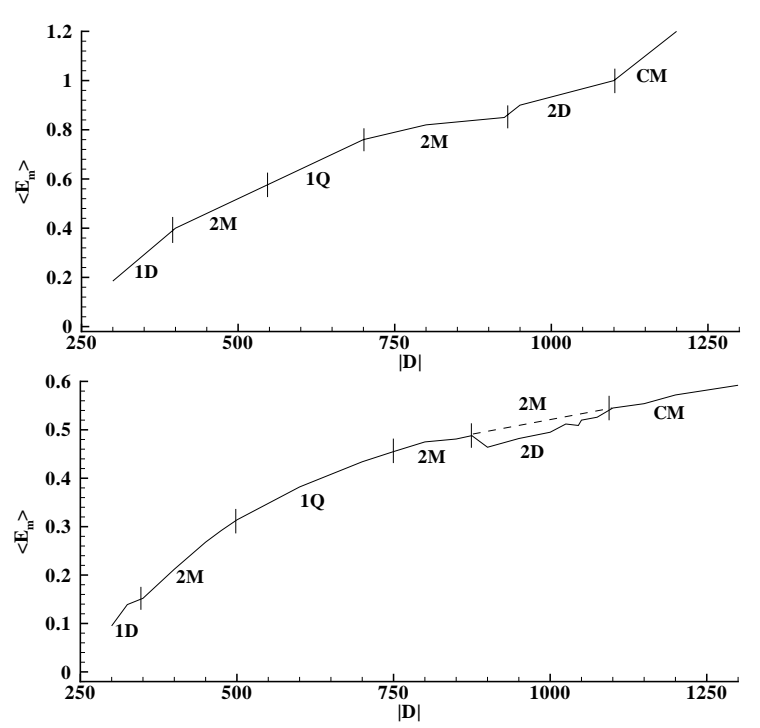

Fig. 2. Bifurcation diagrams determined from the time series presented in T97 (upper panel), and for the solutions summarised here (lower panel). The labels are two-character codes which describe the periodicity and symmetry properties of the solutions (see main text).

and 13 of that paper. The second bifurcation sequence is based on the results we obtained from integrating Eqs. (3)-(5). In each case, the branches are labelled with two-character codes corresponding to the periodicity and parity of the solution. Similar labelling will be used for bifurcation diagrams throughout the rest of this paper. The first character represents the number of oscillation periods present in $E_{\mathrm{m}}$ : either 1,2 , multiple ( $\geq 3$, denoted by $M$ ), or irregular (plausibly chaotic) behaviour (denoted by $\mathrm{C}$ ). The second character describes the symmetry properties of the magnetic field: dipolar (D), quadrupolar (Q), or mixed parity (M).

In general, the two bifurcation sequences are similar. Apart from small discrepancies between the values of $D$ at which the various bifurcations occur, the major difference is that we find two stable solution branches in the parameter regime $875 \lesssim|D| \lesssim 1075$. In contrast, T97 only mentions obtaining pure or strongly dipolar solutions for $900 \lesssim|D| \lesssim 1100$. Given the similarity of the solutions, it is the $P=-1$ branch (solid curve) which should be compared with Tobias' bifurcation sequence. The dashed curve corresponds to an alternative, mixed parity solution branch. It is possible that Tobias also obtained multiple solutions, but only chosen to comment on the dipolar branches. We therefore feel that our basic model can safely be compared with that of T97, although we are using a different numerical technique for solving the equations. This observation is not trivial, since it is possible that different numerical methods may give different results in dynamically fragile regimes.

T97 drew particular attention to solutions for $|D| \gtrsim 1100$. The time series of $E_{\mathrm{m}}, E_{\mathrm{k}}$ and $P$ computed with $D=-1200$ are shown in Fig. 3. In addition to short-term $E_{\mathrm{m}}$ oscillations, there are also long-term modulations driven by the Malkus-Proctor mechanism. Whilst $P \approx-1$ when $E_{\mathrm{m}}$ is large, there are substantial deviations into the mixed parity regime during episodes of reduced activity, which arise from a north/south asymmetry 

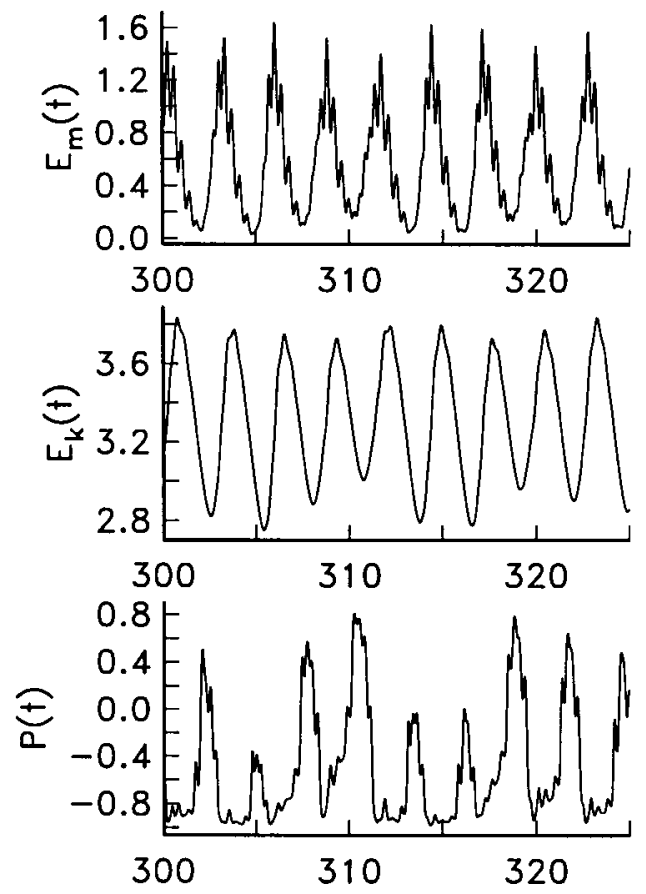

Fig. 3. Time series of $E_{\mathrm{m}}, E_{\mathrm{k}}$ and $P$, computed for the simplest model with $D=-1200$. Besides short-term oscillations, $E_{\mathrm{m}}$ is also modulated on a longer timescale driven by the Malkus-Proctor mechanism. In addition, there are noticeable departures away from $P=-1$ during episodes of reduced magnetic activity. $E_{\mathrm{k}}$ is given in units of $10^{-2}$.

in the magnetic field strength. The time series are qualitatively similar to those presented in Fig. 13 of T97, which were also obtained with $D=-1200$. These features have some likeness to sunspot counts, and T97 classified the behaviour as "solarlike". There are some problems with this idea, which will be outlined in Sect. 4. While the general properties of the time series did not change, computations for larger values of $|D|$ resulted in increasingly irregular behaviour. Given that our code appears to be reliable, we now investigate the behaviour for other, more complex dynamo models.

\section{Modifications to the model}

As noted in Sect. 3 and elsewhere, the time series computed with $D=-1200$ are arguably solar-like. However there are two important problems with this claim. Firstly, if the shortterm $E_{\mathrm{m}}$ oscillations correspond to the sunspot cycle, and the long-term variations to episodes of grand minima, then the only significant fluctuations in $E_{\mathrm{k}}$ occur on a timescale significantly longer than the 11 year period associated with the angular velocity variations in the solar convection zone (Howard \& LaBonte 1980; Howe et al. 2000). This is associated with $\tau \lesssim 1$, and hence an increase in the ratio of hydrodynamic to magnetic timescales. Furthermore, the local maxima of $E_{\mathrm{m}}$ vary greatly from one cycle to the next. This contradicts solar observations, where the changes in maximum sunspot count from cycle to cycle are relatively small.

We are also interested in exploring whether the model described in Sect. 3 (henceforth referred to as the basic model) is truly generic, or if the results change significantly upon making plausible modifications. Helioseismology indicates that gradients in angular velocity are approximately latitudinal in the convection zone, becoming almost radial in the overshoot layer (Thompson et al. 1996; Kosovichev et al. 1997; Schou et al. 1998). It would therefore be sensible to define $U(x, z)$ in terms of a prescribed angular velocity profile, so as to allow the implementation of a more solar-like rotation law. Another concern regarding the basic model is the constraint imposed on $v$ at the lower boundary. Instead of setting $v=0$, it might be more appropriate to impose the stress-free boundary condition $\partial v / \partial z=0$, which is equivalent to conserving angular momentum in spherical geometry. The boundary conditions imposed on $A$ and $B$ are also somewhat arbitrary. However, this issue has already been studied by Brandenburg et al. (1992), Tavakol et al. (1995) and Tworkowski et al. (1998) in spherical geometry, and so we do not pursue it further here.

In contrast to T96 and T97, who interpreted the upper layer as a thin shell above the interface, we take the domain $0<z \leq 1$ to represent the whole of the convection zone. Adopting this definition, both the surface boundary conditions and choice of pole-equator length $(L=4)$ are more physically reasonable. Whilst it is arguably unrealistic to model a "wide" spherical shell as a Cartesian box, Covas et al. (1999) have shown that relatively minor changes to the system geometry do not affect the qualitative nature of the solutions. Interpreting $z=1$ as the solar surface implies that the overshoot layer covers the same radial extent as the convection zone. This is clearly a poor representation of the 1:10 aspect ratio indicated by convection theories and helioseismology. The basic model also features constant $\eta$ throughout the spatial domain. However, toroidal fields are believed to be much stronger in the overshoot layer than in the convection zone, implying a contrast in $\eta$ between the two layers (Parker 1993; Charbonneau \& MacGregor 1997). Inspection of the $B(x, z)$ contours for the basic model (see Sect. 5.2) indicates the field strengths to be comparable. In this regard, defining $\eta$ to be uniform somewhat defeats the object of constructing an interface-type dynamo model.

To test the robustness of the basic model solutions, the four modifications discussed above (stress-free boundary conditions, compressed overshoot layer, non-uniform diffusivity, solar-like rotation law) are made independently. For each of the models, we will determine an extensive (though not necessarily complete) bifurcation sequence, using the same procedure as in Sect. 3. Following that, all of the modifications will be incorporated simultaneously in a "combined" model. Apart from the modifications outlined, the models investigated in Sects. 4.1-4.5 are identical to the basic model.

\subsection{Stress-free boundary conditions}

Setting $\partial v / \partial z=0$ at the upper and lower boundaries has no effect on the critical dynamo numbers. In contrast to the basic model, the bifurcation sequence (shown in Fig. 4) does not feature any doubly periodic, purely dipolar solution branches. Although it is possible that multiple solution branches exist over a narrow range of $|D|$ values, this is perhaps inconsistent with the general "stretching" trend, whereby bifurcations 


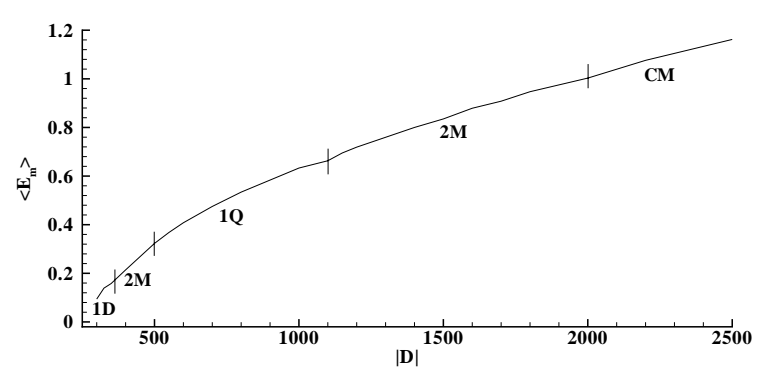

Fig. 4. Bifurcation diagram for the stress-free boundary condition model. Although the overall structure is similar to that for the basic model, the diagram has been "stretched" over a wider range of $|D|$. Also, there are no doubly periodic, dipolar solution branches.

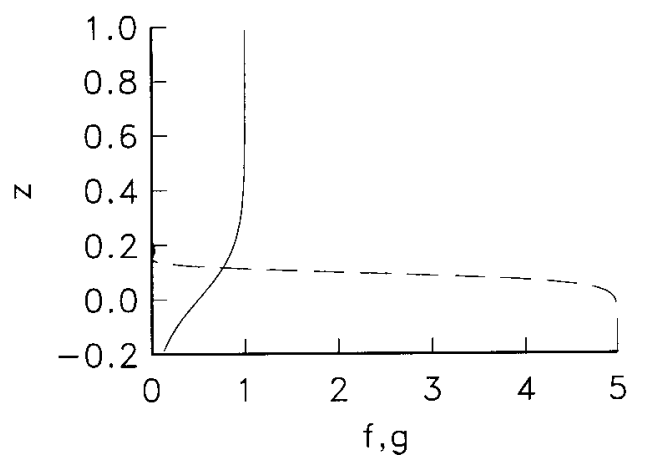

Fig. 5. As for Fig. 1, except for the compressed overshoot layer model. With $z_{1}=-0.2$, the maximum shear is a factor of $a=-1 / z_{1}=5$ larger than for the basic model. With the new definition of $g(z), g \approx 0$ for $z \gtrsim 0.1$ (cf. $z \gtrsim 0.5$ with $z_{1}=-1$ ).

generally occur at more supercritical dynamo numbers (compared to those of Fig. 2). We may therefore conclude that the doubly periodic, dipolar mode either does not exist at all, or is unstable for this particular model. The only significant change in the velocity perturbations was, unsurprisingly, a general increase in $|v(x, z)|$ in the overshoot layer. There is also an overall increase in $|\nabla v|$ throughout the spatial domain.

\subsection{Compressed overshoot layer}

To reduce the width of the overshoot layer whilst retaining the same $\Delta u$ in the domain $z_{1} \leq z<0$, we set

$g(z)= \begin{cases}\frac{a}{2}[1-\tanh 5(a z-0.5)]\left[1-\mathrm{e}^{-25(a z-0.75)^{2}}\right] & z<0.75, \\ 0 & z \geq 0.75,\end{cases}$

where $a=-1 / z_{\text {1. }}$. In order to avoid the need for high resolution and impractically long integration times, we compromise on $z_{1}=-0.2$. As the chosen $\alpha$-effect profile varies mostly in the convection zone, $f(z)$ is left unchanged. Figure 5 shows profiles of $f$ (solid) and $g$ (dashed) for this value of $z_{1}$. Note that $g \approx 0$ in the upper $90 \%$ of the convection zone, compared with only the upper 50\% for the basic model (see Fig. 1). Thus, compressing the overshoot layer leads to the presence of a larger radial shear in the overshoot layer.

The critical dynamo numbers for this model are $D_{\mathrm{c}}(D) \approx$ $D_{\mathrm{c}}(Q) \approx-2959$. Although the definition of the formal nondimensional unit of length is the same as for the basic model,

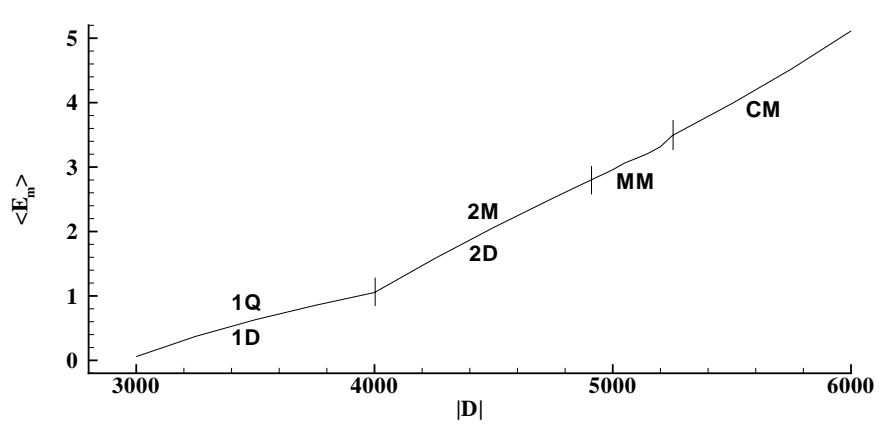

Fig. 6. Bifurcation diagram for the compressed overshoot layer model. In contrast to the basic model bifurcation sequence, some of the solutions exhibit multiple periodicity, and at least two stable solution branches (with comparable $\left\langle E_{\mathrm{m}}\right\rangle$ ) co-exist throughout the explored parameter regime.

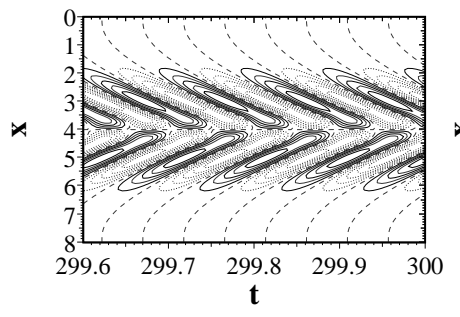

(a) $D=-3000$

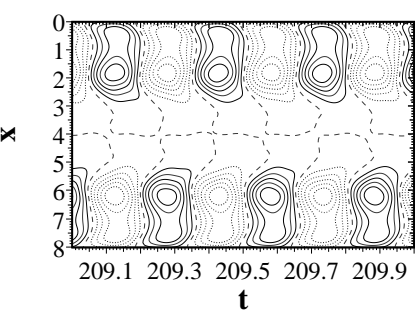

(b) $D=-4500$
Fig. 7. Contours of toroidal field $B(x, t)$ (butterfly diagrams) at a depth of $z_{b}=0.9$, computed for the compressed overshoot layer model with the given values of $D$.

compressing the overshoot layer leads to the presence of smaller length scales in the domain $z_{1} \leq z \leq 0$. Here, the effective dynamo number will be reduced, and a larger "maximum" $|D|$ is therefore required to sustain dynamo action. The close proximity of $D_{\mathrm{c}}(D)$ and $D_{\mathrm{c}}(Q)$ is consistent with the results of Roberts (1972) and Moss et al. (1991a), who found the critical dynamo numbers to be very similar in thin spherical shells, but not in wider shells or spheres.

The bifurcation diagram for this model is shown in Fig. 6. At marginal excitation, integrating the dynamo equations resulted in pure parity, singly periodic behaviour, with $P= \pm 1$ depending on the choice of initial conditions. The co-existence of two stable, oscillatory solution branches is indicative of a pitchfork bifurcation at $D \approx D_{\mathrm{c}}$. Computations in the vicinity of the critical dynamo number failed to reveal any parameter regimes where the final solution state was unique. However, due to the close proximity of $D_{\mathrm{c}}(D)$ and $D_{\mathrm{c}}(Q)$, it is possible that such a regime does exist, but only in an extremely narrow window of $|D|$ close to $D_{\mathrm{c}}$.

Both of the solutions are characterised by a period of oscillation much shorter than the global diffusion time, suggesting that the behaviour is primarily determined by events in the overshoot layer (where length scales are comparitively small). There is also a noticeable reduction in the amplitudes of the $E_{\mathrm{m}}$ and $E_{\mathrm{k}}$ oscillations compared with the basic model. This is presumably a direct consequence of the reduction in cycle 


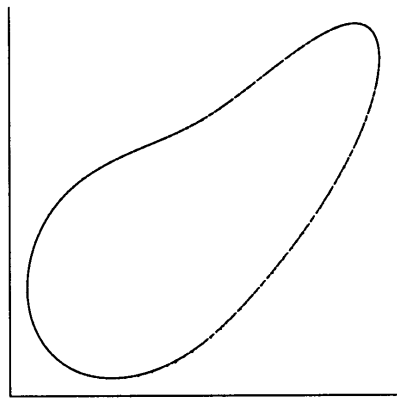

(a) $D=-4500$

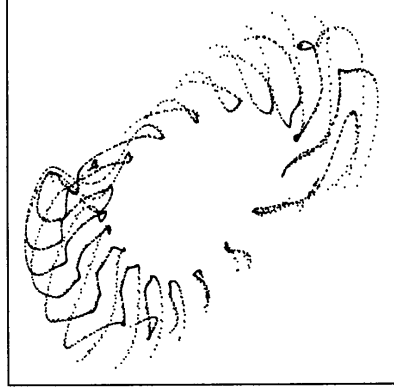

(c) $D=-5200$

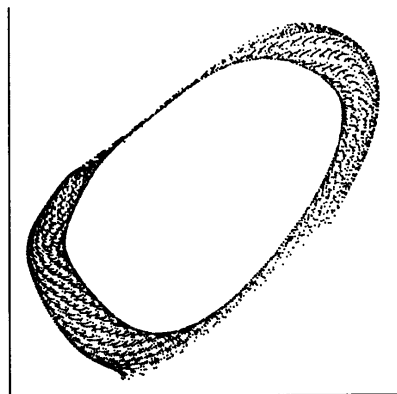

(b) $D=-5100$

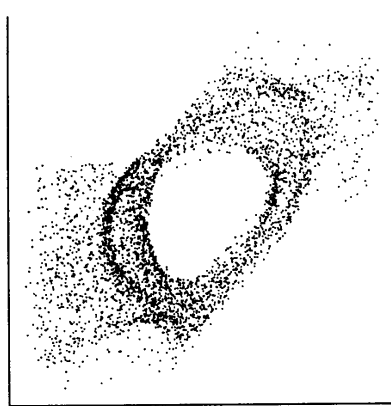

(d) $D=-5250$
Fig. 8. Poincaré maps for the toroidal field at successive phase intervals of $2 \pi$, computed for the compressed overshoot layer model with the given values of $D$. In all these plots, the horizontal axes correspond to values of the vector potential $A\left(L / 2, z_{1} / 2\right)$, the vertical axes give $B(5 L / 8,1 / 2)$, and points are plotted where the value of $B(3 L / 8,0)$ changes sign from positive to negative. (These choices of monitored quantity and points are rather arbitrary, but other choices gave similar results.) The plots are indicative of solutions which are a) doubly periodic, b) multiply (at least triply) periodic, c) multiply periodic with frequency locking, and d) weakly chaotic.

period, and the shorter phases of magnetic (and velocity) field growth and decay. The butterfly diagram corresponding to the dipolar solution obtained with $D=-3000$ (see Fig. 7a) features a large number of active field belts at any one particular time. The migration patterns are similar to those presented in Fig. 12 of Moss \& Brooke (2000), who investigated a spherical shell model with similar features to the one discussed here.

An interesting feature of all the solutions obtained with $4125 \lessgtr|D| \lesssim 5250$ is the presence of at least two stable solutions, characterised by qualitatively similar time series of $E_{\mathrm{m}}$ and $E_{\mathrm{k}}$, and comparable values of $\left\langle E_{\mathrm{m}}\right\rangle$. However, whilst the field is always approximately antisymmetric near the surface (see Fig. 7b), the symmetry properties in the overshoot layer vary substantially depending on the choice of initial conditions. As will be discussed in Sect. 5.1, there is a marked difference in the migration patterns depending on at what depth the field is sampled. For $|D| \gtrsim 5250$, the migrations near the surface were strongly asymmetric, bearing little resemblance to what is observed in the Sun.

The periodicity of the solutions was verified by sampling stroboscopically the phase portraits at successive intervals of $2 \pi$. The Poincaré maps computed with $D=-4500$, $-5100,-5200$ and -5250 (see Figs. 8a-d) show the various transitions (doubly periodic $\rightarrow$ multiply periodic $\rightarrow$ frequency locking $\rightarrow$ chaos). Ruelle \& Takens (1971) showed that motion on a 4-torus (in phase space) is generally unstable. Although Newhouse et al. (1978) later extended this result to a 3-torus, a rather specific type of perturbation was required, and stable motions on 3-tori have since been reported in truncated ODE systems (Tavakol \& Tworkowski 1984). Fourier analysis also indicated the presence of at least three oscillation periods for $4875 \lesssim|D| \lesssim 5250$.

Clearly, compressing the overshoot layer (and shear region) has resulted in a very different solution sequence to that obtained for the basic model. Whereas some of the solutions computed with $z_{1}=-0.2$ feature at least three periods of oscillation, those obtained with $z_{1}=-1$ were either singly periodic, doubly periodic, or irregular. Furthermore, at least two stable solution states were found to co-exist throughout the explored parameter regime (for $|D| \lesssim 5250$ ). In contrast, the bifurcation sequence for the basic model (see Fig. 2) only featured multiple solution branches in a narrow window of $|D|$. In addition, none of the computed solutions resembled that shown in Fig. 3.

\subsection{Non-uniform diffusivity}

We now introduce a "radially" varying diffusivity, $\eta(z)=$ $\left(\eta_{\mathrm{b}}(z)+q\right) /(1+q)$, where $0 \leq \eta_{\mathrm{b}}(z) \leq 1$ is the "base" diffusivity profile

$\eta_{\mathrm{b}}= \begin{cases}0 & z \leq \frac{z_{1}}{2} \\ \frac{1}{2}[1+\tanh 20 z]\left[1-\mathrm{e}^{\left.-80\left(z-\frac{z_{1}}{2}\right)^{2}\right]}\right. & z>\frac{z_{1}}{2},\end{cases}$

and $q=\eta_{\min } /\left(1-\eta_{\min }\right)$, with a minimum diffusivity of $\eta_{\min }$ at the base of the overshoot layer. To avoid numerical difficulties, we choose a modestly small value of $\eta_{\min }=0.2$. Since we wish to investigate the consequences of varying $\eta$ while leaving the other features of the basic model unchanged, we define $z_{1}=-1$, which gives the $\eta(z)$ profile in Fig. 9a. The $\eta(z)$ profile shown in Fig. 9b, computed with $z_{1}=-0.2$, will be introduced in Sect. 4.5.

The critical dynamo numbers are $D_{\mathrm{c}}(D) \approx-115$ and $D_{\mathrm{c}}(Q) \approx-134$. The reduction in $\eta$ in the overshoot layer and the inclusion of turbulent diamagnetism (which transports additional flux from the convection zone) both imply that dynamo action is easier to excite for any given value of $|D|$. Additional computations with the turbulent diamagnetism terms omitted from the MHD dynamo equations indicated an increase in both $D_{\mathrm{c}}(D)$ and $D_{\mathrm{c}}(Q)$. The bifurcation sequence for this model is shown in Fig. 10.

The initial bifurcation is followed by a window of hysteresis, where either pure dipolar or quadrupolar solutions may be obtained depending on the choice of initial conditions. The results presented in this paper suggest that the co-existence of multiple solutions is a not uncommon feature of nonlinear dynamo models. Other examples of complex bifurcation sequences have been provided by Jennings \& Weiss (1991), 


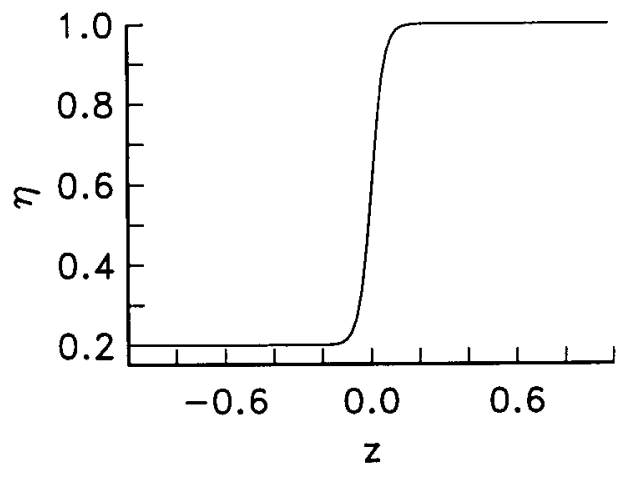

(a) $z_{l}=-1$

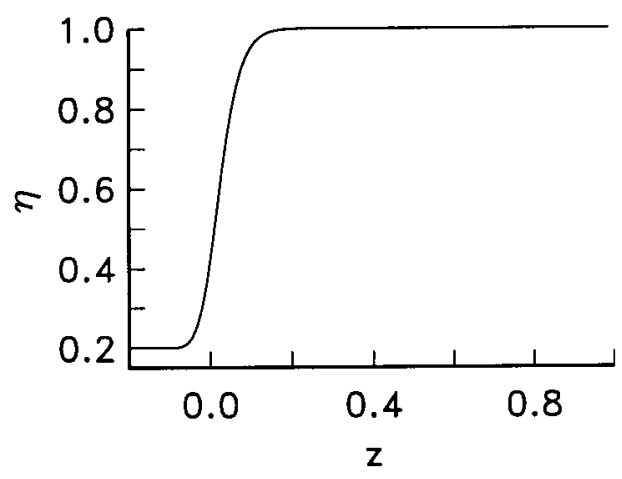

(b) $z_{l}=-0.2$

Fig. 9. Profiles of $\eta(z)$ for the non-uniform diffusivity model, with the lower boundary at a) $z_{1}=-1$, and b) $z_{1}=-0.2$. The diffusivity is largest at the surface, decreasing to some prescribed minimum value $\left(\eta_{\min }=0.2\right)$ in the lower half of the overshoot layer.

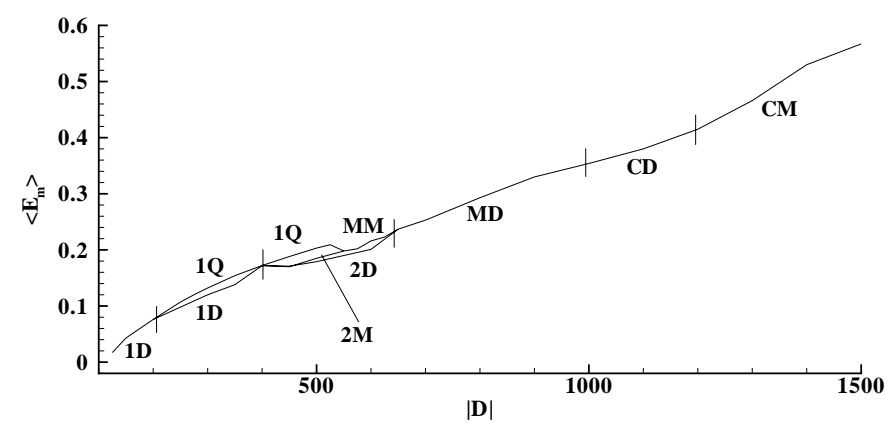

Fig. 10. Bifurcation diagram for the non-uniform diffusivity model. Although some solutions are similar to those for the basic model, the behaviour with non-uniform $\eta$ is generally more complex. In addition to multiply periodic branches, there are also several parameter regimes where at least two stable solutions co-exist.

Brooke \& Moss (1995), Muhli et al. (1995) and Roald \& Thomas (1997). Indeed, three co-existing solution branches, each characterised by qualitatively different behaviour, were found for $450 \lesssim|D| \lesssim 525$. One of these was a doubly periodic, mixed parity mode with long-term modulations occurring on timescales of $t \sim 100$, presumably due to the introduction of longer diffusion times $l^{2} / \eta$ in the overshoot region.

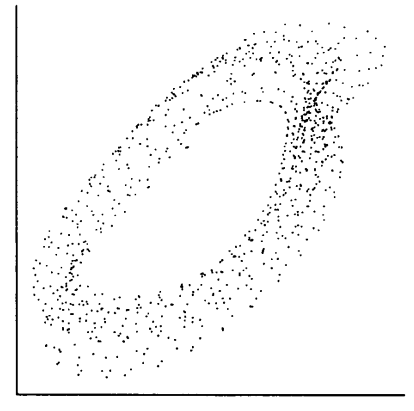

(a) $D=-600$

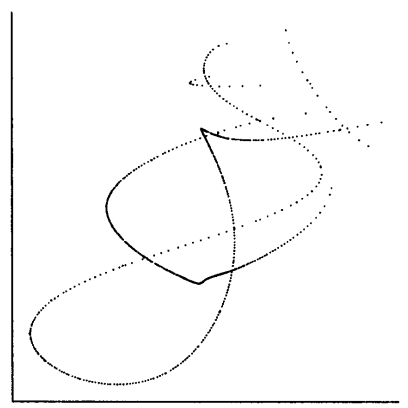

(c) $D=-900$

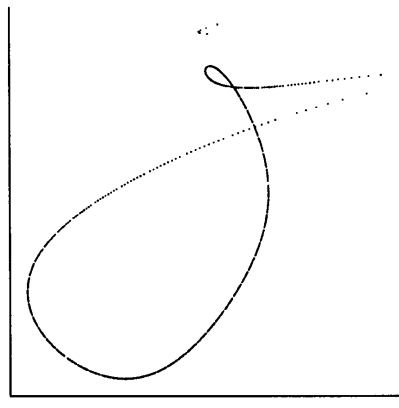

(b) $D=-700$

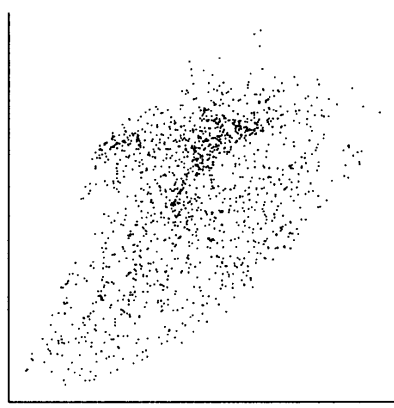

(d) $D=-1100$
Fig. 11. As for Fig. 8, but for the non-uniform diffusivity model. These Poincaré maps are indicative of solutions which are a) multiply periodic, b) approximately doubly periodic with evidence of torus doubling, c) multiply periodic (torus doubled), and d) weakly chaotic.

Inspection of the Poincaré map obtained with $D=-600$ (shown in Fig. 11a) appears to indicate the existence of three $E_{\mathrm{m}}$ oscillation periods, as does Fourier analysis. In the highly supercritical regime, solutions are similar to the doubly periodic behaviour found for previous models, with long-term $E_{\mathrm{m}}$ modulations driven by the Malkus-Proctor mechanism. The essential difference is the presence of a third period twice as long as the long-term modulation, resulting in alternately large and small amplitudes of grand maxima. This is indicative of torus doubling, where a 2-torus in phase space is twisted into a figure of eight and folded back on itself. This phenomenon was first reported in 3D and 4D models of dynamical systems (Kaneko 1983; Arnéodo et al. 1984), and has since been observed by Feudel et al. (1993) in a seventh order system of ODEs designed to model the solar dynamo, and also by Brooke \& Moss (1995), who integrated the MHD dynamo equations numerically in torus geometry. The Poincaré maps computed with $D=-700$ and -900 (see Figs. $11 \mathrm{~b}$ and c) are consistent with this hypothesis.

Since there is evidence for variations in solar activity on intermediate timescales in addition to the 22 year cycle and occurrencies of grand minima (Gleissberg 1967; Garcia \& Mouradian 1998; Pelt et al. 2000), the presence of at least three $E_{\mathrm{m}}$ oscillation periods and episodes of reduced activity is interesting. However, the toroidal field remains strictly antisymmetric about the equator, in contrast to the observed north/south asymmetry fluctuations. As the value of $|D|$ was increased, the 
torus-doubled solution became unstable, leading to irregular oscillations in $E_{\mathrm{m}}$ and $E_{\mathrm{k}}$ with the parity remaining constant at $P=-1$. The Poincaré map computed with $D=-1100$ (shown in Fig. 11d) appears to have no overall structure. Perturbing the solution indicated a strong sensitivity to the initial conditions, providing strong evidence of weakly chaotic behaviour. Further increases in $|D|$ led to even more irregular behaviour, with significant fluctuations in parity and very few intervals where $P \approx-1$.

Whilst some of the computed solutions are similar to those for the basic model (e.g. the doubly periodic, purely dipolar solutions), the behaviour for the non-uniform diffusivity model is generally more complex. In particular, torus doubling was observed at highly supercritical dynamo numbers. In this regime, it is evident that the introduction of a non-uniform diffusivity has led to modulation on longer timescales. This is also true of the mixed parity solutions computed with $450 \lesssim|D| \lesssim 550$, where the periods of the long-term $E_{\mathrm{m}}$ oscillations are of $\sim O(10)$ times longer than those of (otherwise qualitatively similar) solutions obtained for the basic model.

\subsection{Solar-like rotation law}

The angular velocity profile $\Omega(x, z)$ was chosen to be similar to that assumed by Moss (1999). The rotation law in question required some modification since, in the original paper, a spherical polar co-ordinate system was used and attention was restricted to the solar convection zone with no overshoot region. The (modified) angular velocity profile is given by

$\Omega(x, z)= \begin{cases}{[1-h(z)] \Omega_{\mathrm{c}}+h(z) \Omega_{\mathrm{s}}(x)} & z_{1} \leq z \leq 0, \\ \Omega_{\mathrm{s}}(x) & z>0,\end{cases}$

where

$\Omega_{\mathrm{s}}(x)=1-0.126 \cos ^{2}\left(\frac{\pi x}{2 L}\right)-0.159 \cos ^{4}\left(\frac{\pi x}{2 L}\right)$

is the rotation rate at the surface. The inner core is assumed to rotate rigidly with an angular velocity of $\Omega_{\mathrm{c}}=0.96$. The function $h(z)$ is defined as $h=k^{2}(3-2 k)$, where $k=\left(z_{1}-z\right) / z_{1}$. Having prescribed $\Omega$, it is then straightforward to calculate the zero-order velocity $U=\Omega r \sin (\pi x / 2 L)$, where $r(z)=2.33+z$ is the non-dimensionalised radius. The $\alpha$-effect is left in its original form.

For a suitable choice of $z_{1}$, this rotation law is a plausible analytical approximation to those inferred from helioseismology. Since $\Omega_{\mathrm{s}}(x)$ and $\Omega\left(x . z_{1}\right)$ are fixed quantities, compression of the radial shear is automatically taken care of by decreasing the width of the overshoot layer. Figure 12 shows two sets of angular velocity contours, the first for $z_{1}=-1$, and the second for $z_{1}=-0.2$. It is the angular velocity profile in Fig. 12a which will be used in computations throughout the remainder of this section. The rotation law shown in Fig. 12b will be introduced in Sect. 4.5.

The critical dynamo numbers are $D_{\mathrm{c}}(D) \approx-140$ and $D_{\mathrm{c}}(Q) \approx-164$. The reduction in $\left|D_{\mathrm{c}}\right|$ plausibly follows from the introduction of latitudinal differential rotation in regions where the $\alpha$-effect is strong. The bifurcation diagram for this model is shown in Fig. 13. In the moderately supercritical

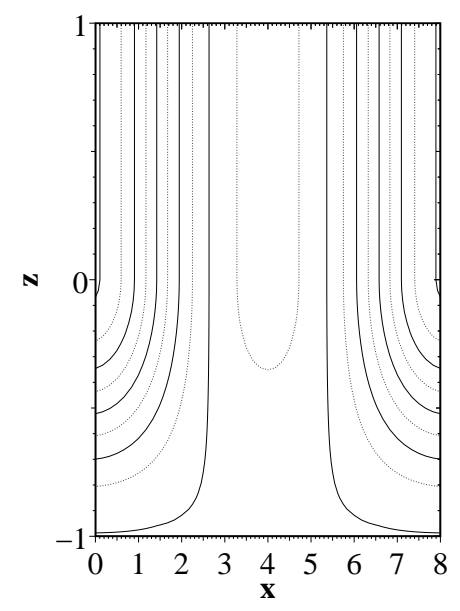

(a) $z_{1}=-1$

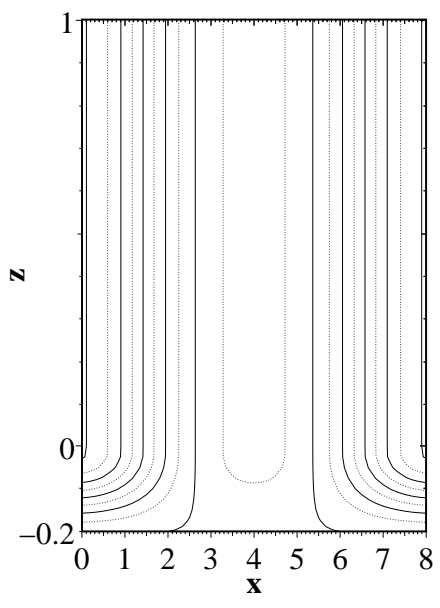

(b) $z_{1}=-0.2$
Fig. 12. Contours of angular velocity $\Omega(x, z)$ for the solar-like rotation model, with the lower boundary at a) $z_{1}=-1$, and b) $z_{1}=-0.2$.

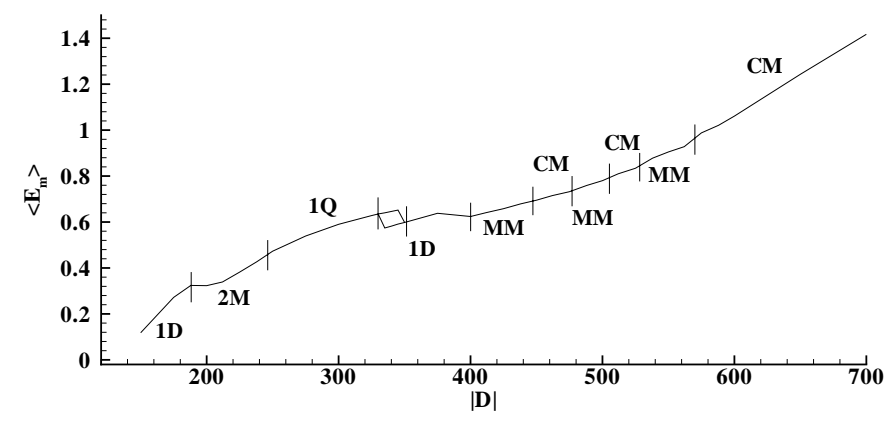

Fig. 13. Bifurcation diagram for the solar-like rotation model. In the marginally supercritical regime, the solutions are similar to those obtained for the basic model. However, the two bifurcation sequences are markedly different for larger values of $|D|$, with alternating windows of multiply periodic and irregular behaviour.

regime, the solution sequence is the same as for the basic model. The agreement ends in a pitchfork bifurcation at $D \approx-335$. As for the non-uniform diffusivity model, there is a window of hysteresis where pure dipolar and quadrupolar solutions co-exist. In this case, the parameter regime is much narrower.

Further increases in $|D|$ lead to a sequence of bifurcations. The existence of alternating windows of periodic and irregular solutions is a well established feature of dynamical systems. Such behaviour has previously been found in MHD dynamo models by Torkelsson \& Brandenburg (1994a,b), Brooke \& Moss (1995), Roald \& Thomas (1997) and Roald (1998). Whilst there are several changes in the solution behaviour throughout the regime $400 \lessgtr|D| \lesssim 600$, the butterfly diagrams are qualitatively similar, with the migration cycle, long-term field strength variations and north/south asymmetry fluctuations generally occurring on different timescales. For $|D| \gtrsim 600$, the behaviour is arguably more interesting, since there are approximately periodic bursts of magnetic energy (characterised by several short-term oscillations) separated by extended intervals where $E_{\mathrm{m}}$ is relatively small. However, there 


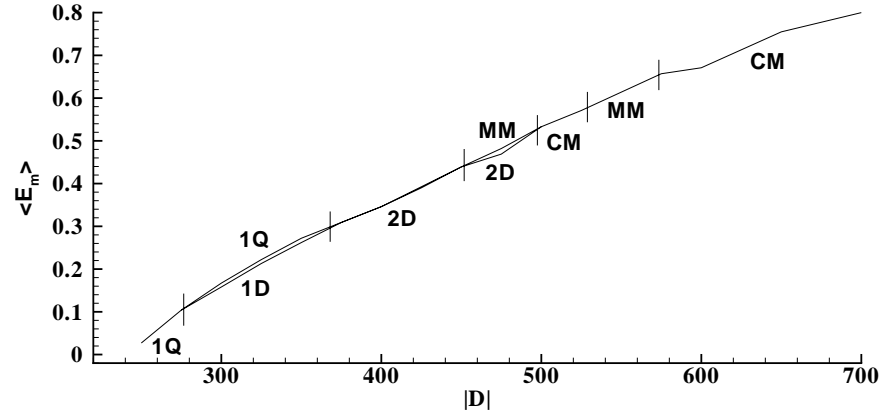

Fig. 14. Bifurcation diagram for the combined model. The solution sequence is quite unlike that determined for the basic model, featuring an initial bifurcation to a pure quadrupolar mode, several co-existing multiple solution branches, and a series of torus doubled solutions before a transition to irregular behaviour.

are also substantial parity variations, with only limited intervals where $P \approx-1$.

The similarity with the basic model bifurcation sequence in the regime of marginal supercriticality suggests that, provided $|D|$ is not too large, radial variations in $\Omega$ in the overshoot layer play a more important rôle in determining the solution behaviour than latitudinal variations in the convection zone. This hypothesis is supported by the persistence of latitudinally (as opposed to radially) migrating belts of toroidal field (consistent with dynamo waves propagating along contours of constant $\Omega$, cf. Yoshimura 1975). Comparing profiles of $|\partial \Omega / \partial x|$ and $|\partial \Omega / \partial z|$ for the two models indicates the latitudinal gradients to be relatively small, even for $z_{1}=-1$. It is worth noting that the change in (equatorial) zero-order velocity $\Delta U$ across the respective shear regions are comparable. At more supercritical values of $|D|$, the latitudinal shear arguably makes a significant contribution to toroidal field generation, and the nature of the solutions can be expected to differ. Once again, the "solar-like" solutions obtained for the basic model are not robust.

\subsection{A "combined" model}

Having investigated the effects of the individual modifications, we now combine the features into one model. As in Sect. 4.2, we set $z_{1}=-0.2$. Adopting the same definitions of $\eta(z)$ and $\Omega(x, z)$ as in Sects. 4.3 and 4.4 gives the magnetic diffusivity profile in Fig. 9b, and the rotation law in Fig. 12b. The MHD dynamo equations are solved subject to boundary conditions defined in Sect. 3, except with stress-free constraints imposed at the lower boundary, i.e. $\partial v / \partial z=0$. The critical dynamo numbers were found to be $D_{\mathrm{c}}(D) \approx-243$ and $D_{\mathrm{c}}(Q) \approx-242$.

The bifurcation diagram for this model is shown in Fig. 14. The initial quadrupolar branch is followed by a window of hysteresis, where pure dipolar and quadrupolar solutions co-exist. Multiple solution branches also occur in the parameter regime $450 \lesssim|D| \lesssim 500$. Time series of $E_{\mathrm{m}}, E_{\mathrm{k}}$ and $P$ for the multiply periodic solution computed with $D=-475$ are shown in Fig. 15. Whilst $P \approx-1$ when $E_{\mathrm{m}}$ is large, there are small $(\Delta P \approx 0.03)$ deviations from the pure parity state during grand minima in activity. The pattern of behaviour resembles that of
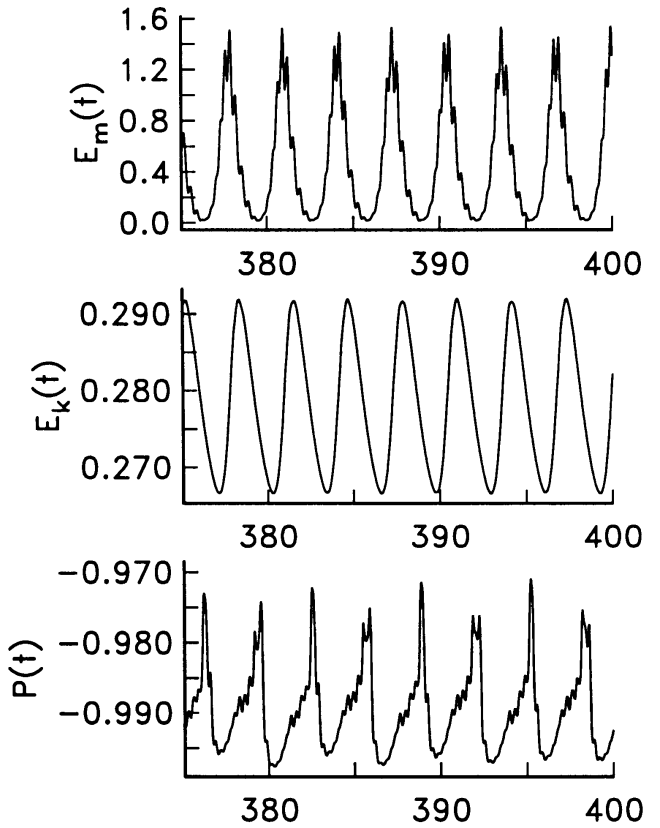

Fig. 15. Time series of $E_{\mathrm{m}}, E_{\mathrm{k}}$ and $P$, computed for the combined model with $D=-475$. In some respects, the solution is arguably solar-like, featuring short-term $E_{\mathrm{m}}$ oscillations and deviations away from $P=-1$ during episodes of reduced activity.

the basic model solution shown in Fig. 3, except with more regular fluctuations in energy and parity. The corresponding Poincaré map forms an unbroken limit cycle with two distinct orbits, indicating torus doubling. Although the grand maxima in $E_{\mathrm{m}}$ in Fig. 15 appear to have similar amplitudes, there are noticeable differences between the magnitudes of the parity deviations.

The time series computed with $D=-500$ are shown in Fig. 16. Although there is a noticeable torus doubling effect (note the alternating sizes of grand maxima), the oscillations are no longer regular. The magnetic field is approximately symmetric when $E_{\mathrm{m}}$ is large, whilst episodes of reduced activity are correlated with seemingly irregular departures in parity away from $P=+1$. Sometimes, the deviations are so large that $P \approx-1$. During these episodes, $\left\langle E_{\mathrm{m}}\right\rangle$ becomes noticeably smaller. This behaviour is consistent with the description of "icicle" intermittency given by Brooke et al. (1998). Following a bifurcation at $D \approx-525$, the solutions are multiply periodic. Instead of irregular, "icicle" type fluctuations, the parity varies smoothly, with an oscillatory period equal to that of the longterm modulations. Contrary to what is observed in the Sun, there are only a few intervals where $P \approx-1$, which occur as the solution enters a state of grand minima. Taking $|D| \gtrsim 575$ results in irregular, arguably weakly chaotic, solutions with substantial fluctuations in parity.

In some respects, the bifurcation sequence is similar to that for the non-uniform diffusivity model, with an initial bifurcation to a singly periodic, pure parity mode, followed by a reasonably large window of hysteresis. Both bifurcation sequences also contain a branch of doubly periodic, dipolar solutions with long-term $E_{\mathrm{m}}$ modulations driven by the Malkus-Proctor mechanism. Further increases in $|D|$ lead to torus doubling. However, 

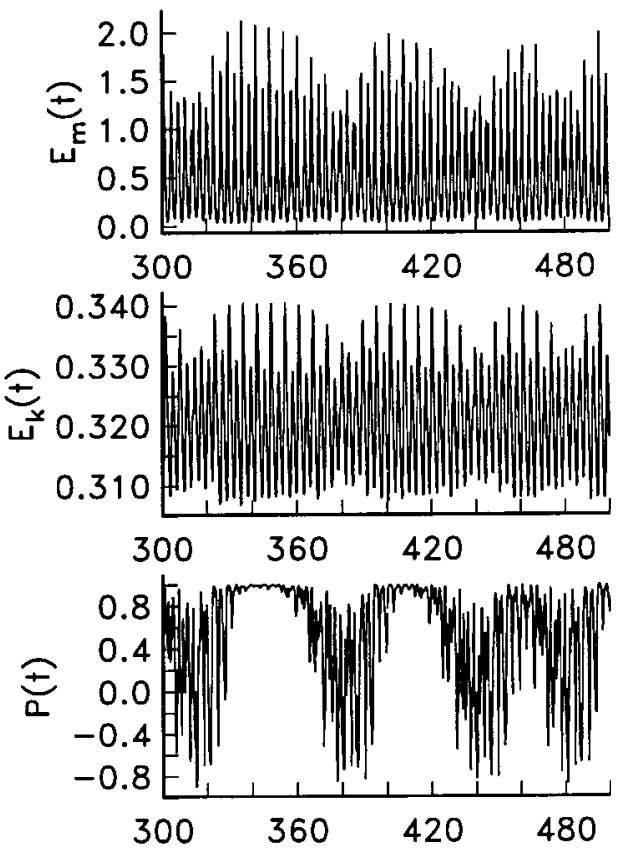

Fig. 16. As for Fig. 15, except with $D=-500$. The $E_{\mathrm{m}}$ oscillations are irregular, with episodes of reduced activity correlated with deviations in parity away from $P=+1$. The overall pattern of behaviour matches the description of "icicle" intermittency given by Brooke et al. (1998).

the combined model solutions do not remain strictly dipolar in the regime of high supercriticality. Consistent with findings for the solar-like rotation model, there are large fluctuations in parity, with a window of chaotic behaviour "sandwiched" between two regular solution branches.

Despite the close proximity of $D_{\mathrm{c}}(D)$ and $D_{\mathrm{c}}(Q)$, compressing the overshoot layer did not produce any co-existing solution branches with indistinguishable $E_{\mathrm{m}}$ and $E_{\mathrm{k}}$ time series. Given the presence of strong "radial" gradients in $\Omega$ in the overshoot layer, we may plausibly have expected to observe similarities with the bifurcation sequence in Fig. 6 , at least for $D \approx D_{\text {c }}$. However, with $z_{1}=-0.2$, the value of $\Delta U$ across the shear region is several times larger for the compressed overshoot layer model. Since the velocity perturbations are somewhat dependent on the assumed rotation law, it is not surprising that the trends reported in Sect. 4.1 (horizontal "stretching" of the bifurcation diagram, absence of doubly periodic dipolar solutions) do not occur in the combined model.

\section{Field structures and migration patterns}

\subsection{Variations in oscillation period with depth}

Typical contours of $B(x, z)$ computed at marginal supercriticality $(D=-3000)$ for the compressed overshoot layer model are shown in Fig. 17a. In contrast to the field patterns obtained for the basic model, most of the toroidal flux is concentrated in the region $z \lesssim 0.5$. As noted previously, setting $z_{1}=-1 \mathrm{im}$ plies that the velocity shear $g \approx 0$ for $z \gtrsim 0.5$, whereas setting $z_{1}=-0.2$ gives $g \approx 0$ for $z \gtrsim 0.1$. It is therefore not surprising

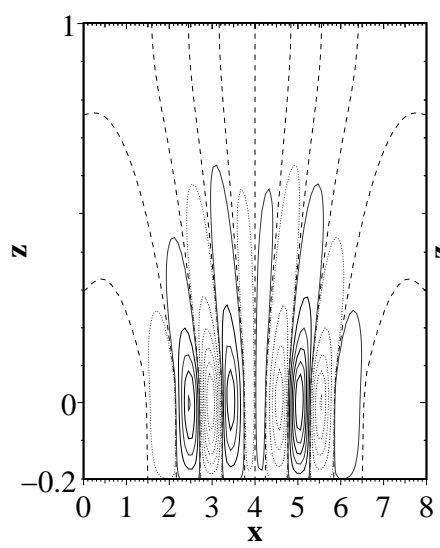

(a) $D=-3000$

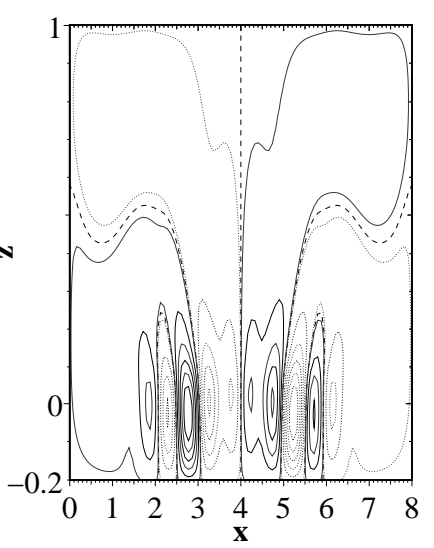

(b) $D=-4500$
Fig. 17. Typical contours of toroidal field $B(x, z)$, computed for the compressed overshoot layer model with given values of $D$. In both cases, strong fields are produced in the overshoot layer, characterised by cells of narrow latitudinal extent. In case b), some of these cells merge together midway through the convection zone to form larger cells near the surface.

that the fields now observed are considerably weaker near the solar surface than in the overshoot layer.

In addition to strong toroidal fields, the contour plot is also characterised by "cells" of narrow latitudinal extent, consistent with a short cycle period. The field patterns are similar to those presented in Fig. 11a of Moss \& Brooke (2000), who performed a limited number of computations for a comparable model, but in spherical geometry. Large numbers of cells were also reported by Moss et al. (1990) and Tavakol et al. (1995) in models featuring thin "overshoot" layers. For solarlike angular velocity profiles (cf. Fig. 12b), comparatively few cells tend to be present. However, similar field structures were found by Rüdiger \& Brandenburg (1995) in a model featuring anisotropic $\alpha$ and $\eta$, a solar-like rotation law and two nonlinearities ( $\alpha$-quenching and magnetic buoyancy).

As noted in Sect. 4.2, migration patterns at more supercritical dynamo numbers $(|D| \gtrsim 4000)$ can be markedly different depending on at what depth the field is sampled. Figure $17 \mathrm{~b}$ shows typical contours of $B(x, z)$ computed with $D=-4500$, and butterfly diagrams for a selection of depths are shown in Figs. 18a-f. Depending on the phase of the cycle and the polarity of the field, the narrow cells produced in the shear region are either isolated from the surface or merge together to form larger cells midway through the convection zone. At the surface, there is generally only one migratory field belt per hemisphere at any one time, whilst there are up to five near the interface. The concentration of flux at mid-latitudes in the upper convection zone is presumably due to longer diffusion times, which result in slower migration. Another possible reason is the chosen form of the latitudinal dependence (i.e. dependence on $x$ ) of the $\alpha$-effect, which is strongest near the poles: other authors (e.g. Rüdiger \& Brandenburg 1995; Moss \& Brooke 2000) have experimented with different latitudinal dependences for alpha. 


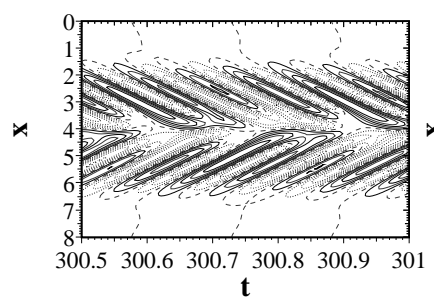

(a) $z_{b}=0$

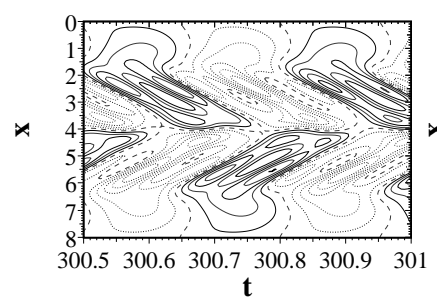

(c) $z_{b}=0.3$

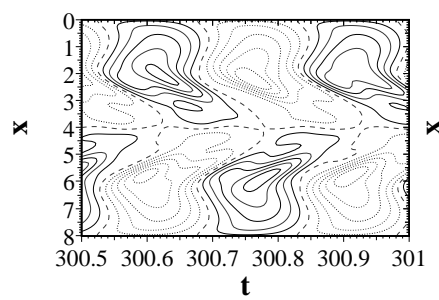

(e) $z_{b}=0.5$

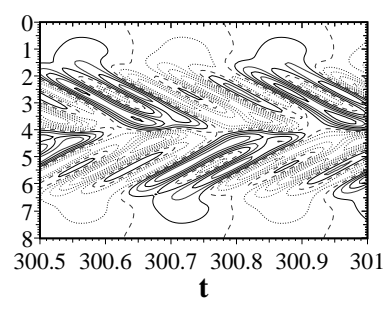

(b) $z_{b}=0.2$

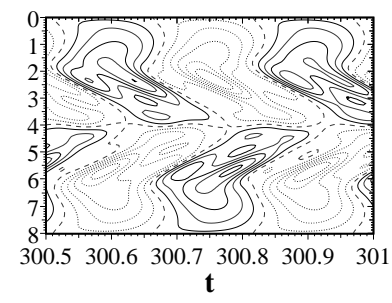

(d) $z_{b}=0.4$

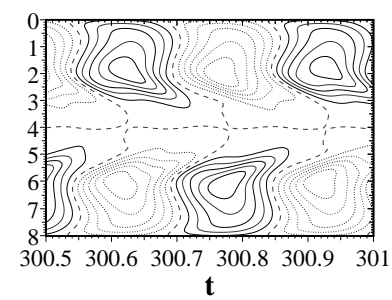

(f) $z_{b}=0.6$
Fig. 18. Contours of toroidal field $B(x, t)$ (butterfly diagrams) computed at given depths for the compressed overshoot layer model with $D=-4500$.

A number of studies have suggested that sunspots are the surface manifestations of flux tubes formed in the strong-field region near the base of the convection zone, which rise to the surface via some sort of MHD instability. If this is the case, and models with a cell-like structure near the interface, similar to those we have discussed, are applicable, then the cell structure must be lost during the rise. We note however that simulations (e.g. Brandenburg et al. 1996) suggest that there may be a significant downward pumping of flux into the overshoot region, which might oppose the flux tube rise. Also, we note that a number of earlier investigations have published butterfly diagrams generated from the local toroidal field near the surface of the model. Remember additionally that if flux tubes do rise from the base of the $\mathrm{CZ}$ to the surface, then there may be a significant latitudinal displacement during the rise; in that case there will not be a direct correspondence between toroidal field migration patterns at any depth and the observed butterfly diagram. At the least we deduce that the choice of depth needs to be made with some care.

\subsection{Storage of magnetic flux in the overshoot layer}

One of the main results obtained by Parker (1993) was the relationship $B_{\mathrm{ov}}{ }^{2} / B_{\mathrm{cz}}{ }^{2} \sim \eta_{\mathrm{cz}} / \eta_{\mathrm{ov}}$, where $B_{\mathrm{ov}}$ and $B_{\mathrm{cz}}$ are the
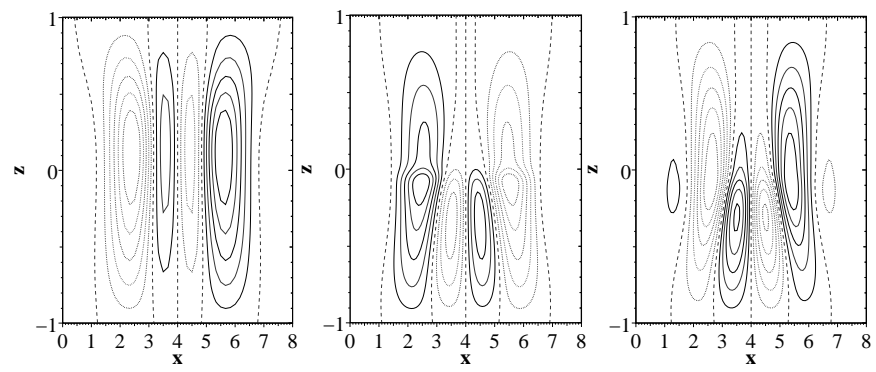

Fig. 19. Typical contours of toroidal field $B(x, z)$, computed at marginal supercriticality for the basic model (left panel), the nonuniform diffusivity model (centre panel), and for the same case except without turbulent diamagnetism (right panel). Here, the lower boundary was set to $z_{1}=-1$.

maximum toroidal field strengths in the overshoot layer and convection zone, and $\eta_{\mathrm{ov}}$ and $\eta_{\mathrm{cz}}$ are the corresponding magnetic diffusivities. Typical contours of $B(x, z)$ computed for the basic model with $D=-300$ (marginally supercritical) are shown in the left panel of Fig. 19. As expected, toroidal field strengths in the overshoot layer are comparable with those in the convection zone. Although this is consistent with Parker's relationship, numerical simulations indicate that $B_{\mathrm{ov}} \gg B_{\mathrm{cz}}$ in the actual Sun, which implies some contrast in diffusivity.

Typical contours of $B(x, z)$ computed for the non-uniform diffusivity model with $D=-150$ are shown in the centre panel of Fig. 19. Here, toroidal field strengths in the overshoot layer are generally greater than in the convection zone, with estimates suggesting $B_{\mathrm{ov}} / B_{\mathrm{cz}} \approx 1.87$. Some discrepancy with the predicted ratio of $\sqrt{5} \approx 2.24$ is to be expected, since Parker's model is discontinuous at the interface, with discrete values of $\eta$ in the two layers. Here, $\eta$ varies smoothly from $\eta_{\min }$ to unity, with a steep (but continuous) gradient at $z \approx 0$. MacGregor \& Charbonneau (1997) constructed a model with the $\alpha$-effect and shear layers separated by a region where the only important process was turbulent diffusion. For their model, the predicted field strength ratio is $B_{\mathrm{ov}} / B_{\mathrm{cz}} \sim \eta_{\mathrm{cz}} / \eta_{\mathrm{ov}}=5$. As separating the layers leads to an increase in the field strength ratio, it seems somewhat intuitive that modelling the $\alpha$-effect and $\omega$ effect as continuous functions with a region of overlap should lead to a reduction in $B_{\mathrm{ov}} / B_{\mathrm{cz}}$ compared with Parker's model. Computations with $\eta_{\min }=0.01$ gave field strength ratios of $B_{\mathrm{ov}} / B_{\mathrm{cz}} \sim 10$.

To investigate the importance of turbulent diamagnetism, an additional computation was performed with the relevant terms omitted from the dynamo equations. Contours of $B(x, z)$ computed with $D=-200$ are shown in the right panel of Fig. 19. Although faster ohmic decay leads to a concentration of toroidal flux in the overshoot layer towards the end of each migratory cycle, the inclusion of turbulent diamagnetism clearly makes a significant contribution to the field distribution at mid-latitudes. With this feature "switched off", $B_{\mathrm{ov}} \sim B_{\mathrm{cz}}$. Thus, it appears that Parker's relationship may not, in general, be extended to models with continuous profiles of $\alpha, \Omega$ and $\eta$. These results suggest that some mechanism for the preferential downward transport of magnetic flux, such as 

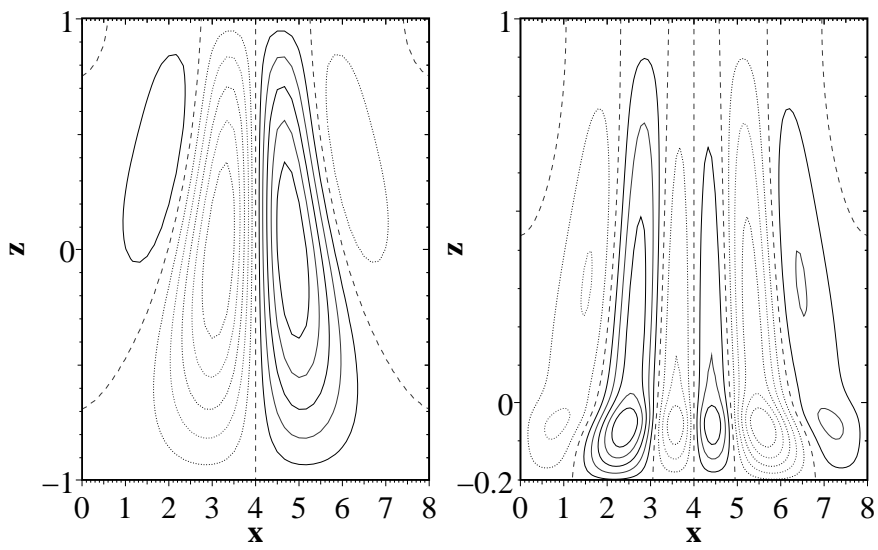

Fig. 20. Typical contours of toroidal field $B(x, z)$, computed for the solar-like rotation model with $D=-150$ (left panel), and for the combined model with $D=-300$ (right panel).

turbulent diamagnetism or magnetic pumping (Nordlund et al. 1992; Brandenburg et al. 1996; Tobias et al. 1998, 2001), is an essential component of interface-type dynamo models.

\subsection{Region of toroidal field generation}

Although it is not clear whether the toroidal field belts (i.e. sunspots) observed at the solar surface are produced in the convection zone or overshoot layer, convincing arguments suggest the latter to be the case (e.g. Spiegel \& Weiss 1980; Schmitt \& Rosner 1983; Moreno-Insertis et al. 1992). Typical contours of $B(x, z)$ computed at marginal supercriticality $(D=-150)$ for the solar-like rotation model (see the left panel of Fig. 20) show surface fields originating in the convection zone. Based on results presented in Sects. 5.1 and 5.2, it is plausible that this inconsistency will be resolved by compressing the overshoot layer and/or introducing a contrast in $\eta$ with turbulent diamagnetism. Typical contours of $B(x, z)$ computed for the combined model with $D=-300$ are shown in the right panel of Fig. 20. Despite the decrease in cycle period, there are no large or isolated cells such as those in Fig. 17b. However, the effects of turbulent diamagnetism are clearly visible. Although some toroidal flux is generated in the convection zone (note the cells in the polar regions), this tends to occur fairly deep beneath the surface. Furthermore, once the field belts have migrated to low latitudes, a significant proportion of the flux is found in the overshoot layer.

\section{Discussion and conclusions}

Our main purpose in this paper is to address the question of whether a generic bifurcation sequence exists for interface models that is qualitatively robust to plausible changes in the model, provided that major dynamical factors such as the form of the nonlinear feedback mechanism are not changed. Our main finding is that the bifurcation sequences computed for interface-type dynamo models do not appear to be robust to plausible changes in aspect ratios and physical parameters in the convective and overshoot regions.
A modest change in the boundary conditions leads to an entire branch of solutions becoming unstable. More dramatic departures in solution behaviour are observed following compression of the overshoot layer, the introduction of non-uniform magnetic diffusivity, and the implementation of a solar-like rotation law. The solutions appear to be particularly sensitive to changes in the angular velocity profile. The form of the initial bifurcation appears to be important in determining the structure of the full bifurcation sequence. Specifically, different behaviour is obtained when the quadrupolar and dipolar solutions bifurcate from the trivial solution at almost the same critical values, in contrast to the basic model where the dipolar solution bifurcates at a considerably lower critical value. This is related to the formation of many cells of migrating waves in the overshoot region, so that the structure of the unsigned quadrupolar and dipolar fields is very similar. Additionally, we have shown that the behaviour in the convection zone is important in determining whether this complexity is observed at the surface and this is highly model dependent.

We do not conclude from this that low-order models have no value. On the contrary, they are of great importance in understanding the behaviour at the bifurcations observed in higher order numerical models. An example of this is the model of Knobloch \& Landsberg (1996), which considers the situation when the dynamics is determined by the bifurcation of quadrupolar and dipolar modes occurring at almost exactly the same critical value; these modes have spatial structures that are identical except for sign about the equator. This model predicts very rich dynamical behaviour, important aspects of which are confirmed in the numerical models of Sect. 4.2, where the overshoot region is compressed relative to the convective zone. Their low-order model gives deeper insight into the possible dynamics of this near-double bifurcation, since the restriction in structure of the equations makes a more fully comprehensive analysis possible. The detailed numerical investigations are, however, essential in ascertaining which bifurcations are observed in different physical models, and thus the appropriate lower order models to be studied.

One very important theme that emerges from our investigations is the possible difference in structure of the migrating wave patterns at differing depths. We note that, in spherical geometry, Covas et al. (2000) have described a general framework for such behaviour which they call "spatiotemporal fragmentation", and demonstrated its occurrence in the contours of the perturbation velocity field. In our investigations, it appears in the magnetic field contours, but it is a feature which is model dependent. For example, it appears in the compressed overshoot region model of Sect. 4.2 but not in the combined model of Sect. 4.5. We stress that this has important implications for the comparison of dynamo models with the observed solar activity cycle, raising the question as to whether we are observing activity tracers of magnetic waves in the overshoot layer or much closer to the surface in the convection zone.

Our results show that the coupling of behaviour between the convective and overshoot regions is of critical importance for the dynamical behaviour of the solution, and must be modelled as carefully as possible. The findings of more sophisticated observational techniques should therefore be 
incorporated into dynamo models, especially with regards to the large-scale velocity fields present in the Sun (e.g. rotation law, meridional circulation, giant convective cells).

Including the effects of meridional circulation can lead to solar-like migration patterns with $D>0$ (Choudhuri et al. 1995; Dikpati \& Charbonneau 1999; Dikpati \& Gilman 2001; Küker et al. 2001). However, this requires quite specific and restrictive profiles of the $\alpha$-effect and magnetic diffusivity (e.g. Phillips 2001). Given the apparent necessity to include some mechanism for the preferential downward transport of magnetic flux, it may be important to model the $\alpha$-effect and diffusivity as anisotropic tensors (e.g. Kitchatinov \& Rüdiger 1993, 1995). Although numerical simulations suggest that the components of the $\alpha$-tensor have different radial and latitudinal dependencies (e.g. Brandenburg et al. 1990; Krivodubskii 1998; Ossendrijver et al. 2001), it is not obvious what form the anisotropies should take.

We did not find coherent torsional oscillations in the calculations described here. This appears to be because of the relatively small value adopted for the turbulent Prandtl number $\left(P_{\mathrm{r}}=0.1\right)$, necessary to produce "interesting" dynamical behaviour. Further work (Brooke et al., in preparation), and also e.g. Covas et al. (2001) suggests that Prandtl numbers of order unity are require to produce solar-like torsional oscillations. This Prandtl number dilemma is discussed in more detail in Brooke et al. (in preparation).

Due to its relative proximity to the Earth, considerably more observational data is available for the Sun than for other stars. While studies of other main sequence stars have revealed cyclic variations in magnetic activity, and inferred the occurrence of grand minima (Baliunas et al. 1995), their rotation laws (other than basic rotation periods) are unknown. Since the form of the rotation law is important for the behaviour of the dynamo, both theoretical studies of stellar rotation and results from improved techniques of asteroseismology will be of importance. For this reason alone, it is important to continue to investigate dynamo models with a variety of rotation laws and other details of the dynamo generating regions, since we do not fully understand how typical the current solar configuration is, nor how constant it has been throughout the Sun's history.

Acknowledgements. AP acknowledges support received under the Endowment Scholarship Scheme of the Faculty of Science and Engineering of the University of Manchester.

\section{References}

Arnéodo, A., Coullet, P. H., \& Spiegel, E. A. 1984, Phys. Lett., A 94, 1 Baliunas, S. L., et al. 1995, ApJ, 438, 269

Blackman, E. G., \& Field, G. B. 1999, ApJ, 521, 597

Blackman, E. G., \& Field, G. B. 2000, ApJ, 534, 984

Brandenburg, A. 2001, ApJ, 550, 824

Brandenburg, A., \& Donner, K. J. 1997, MNRAS, 288, L29

Brandenburg, A., Jennings, R. L., Nordlund, Å., et al. 1996, J. Fluid Mech., 306, 325

Brandenburg, A., Moss, D., \& Tuominen, I. 1992, A\&A, 265, 328

Brandenburg, A., Nordlund, Å., Pulkkinen, P., Stein, R. F., \& Tuominen, I. 1990, A\&A, 232, 277

Brooke, J. M., \& Moss, D. 1994, MNRAS, 266, 733
Brooke, J. M., \& Moss, D. 1995, A\&A, 303, 307

Brooke, J. M., Pelt, J., Tavakol, R., \& Tworkowski, A. 1998, A\&A, 332, 339

Caligari, P., Moreno-Insertis, F., \& Schüssler, M. 1995, ApJ, 441, 886

Caligari, P., Schüssler, M., \& Moreno-Insertis, F. 1998, ApJ, 502, 481

Cattaneo, F., \& Hughes, D. W. 1996, Phys. Rev., E54, R4532

Charbonneau, P., \& MacGregor, K. B. 1997, ApJ, 486, 502

Choudhuri, A. R., Schüssler, M., \& Dikpati, M. 1995, A\&A, 303, L29

Covas, E., Tavakol, R., Tworkowksi, A., et al. 1999, A\&A, 345, 669

Covas, E., Tavakol, R., \& Moss, D. 2000, A\&A, 363, L13

Covas, E., Tavakol, R., \& Moss, D. 2001, A\&A, 371, 718

Dikpati, M., \& Charbonneau, P. 1999, ApJ, 518, 508

Dikpati, M., \& Gilman, P. 2001, ApJ, 559, 428

Fan, Y., Fisher, G. H., \& DeLuca, E. E. 1993, ApJ, 405, 390

Feudel, U., Jansen, W., \& Kurths, J. 1993, Int. J. Bifur. Chaos, 3, 131

Garcia, A., \& Mouradian, Z. 1998, Sol. Phys., 180, 495

Gleissberg, W. 1967, Sol. Phys., 2, 231

Guckenheimer, J., \& Holmes, P., Nonlinear Oscillations, Dynamical Systems and Bifurcations of Vector Fields (Springer-Verlag, New York), 1983

Howard, R., \& LaBonte, B. J. 1980, ApJ, 239, L33

Howe, R., Christensen-Dalsgaard, J., Hill, F., et al. 2000, ApJ, 533, L163

Jennings, R. L., \& Weiss, N. O. 1991, MNRAS, 252, 249

Kaneko, K. 1983, Prog. Theo. Phys., 69, 1806

Kitchatinov, L. L., \& Rüdiger, G. 1993, A\&A, 276, 96

Kitchatinov, L. L., \& Rüdiger, G. 1995, A\&A, 299, 446

Knobloch, E., \& Landsberg, A. S. 1996, MNRAS, 278, 294

Knobloch, E., Tobias, S. M., \& Weiss, N. O. 1998, MNRAS, 297, 1123

Kosovichev, A. G., et al. 1997, Sol. Phys., 170, 43

Krivodubskii, V. N. 1998, Astron. Rep., 42, 122

Küker, M., Rüdiger, G., \& Schultz, M. 2001, A\&A, 374, 301

MacGregor, K. B., \& Charbonneau, P. 1997, ApJ, 486, 484

Malkus, W. V. R., \& Proctor, M. R. E. 1975, J. Fluid Mech., 67, 417

Markiel, J. A., \& Thomas, J. H. 1999, ApJ, 523, 827

Moffatt, H. K. 1978, Magnetic field generation in electrically conducting fluids (Cambridge Univ. Press, Cambridge)

Moreno-Insertis, F. 1983, A\&A, 122, 241

Moreno-Insertis, F., Schüssler, M., \& Ferriz-Mas, A. 1992, A\&A, 264 686

Moss, D. 1999, MNRAS, 306, 300

Moss, D., Tuominen, I., \& Brandenburg, A. 1991a, A\&A, 245, 129

Moss, D., Brandenburg, A., \& Tuominen, I. 1991b, A\&A, 247, 576

Moss, D., \& Brooke, J. 2000, MNRAS, 315, 521

Moss, D., Tuominen, I., \& Brandenburg, A. 1990, A\&A, 240, 142

Muhli, P., Brandenburg, A., Moss, D., \& Tuominen, I. 1995, A\&A, 296, 700

Newhouse, S., Ruelle, D., \& Takens, F. 1978, Comm. Math. Phys., 64, 35

Nordlund, Å., Brandenburg, A., Jennings, R. L., et al. 1992, ApJ, 392, 647

Ossendrijver, M., Stix, M., \& Brandenburg, A. 2001, A\&A, 376, 713

Parker, E. N. 1975, ApJ, 198, 205

Parker, E. N. 1979, Cosmical magnetic fields (Clarendon Press, Oxford)

Parker, E. N. 1993, ApJ, 408, 707

Pelt, J., Brooke, J., Pulkkinen, P. J., \& Tuominen, I. 2000, A\&A, 362, 1143

Phillips, A. D. 2001, Ph.D. Thesis, University of Manchester

Ribes, J. C., \& Nesme-Ribes, E. 1993, A\&A, 276, 549

Roald, C. B. 1998, MNRAS, 300, 397

Roald, C. B., \& Thomas, J. H. 1997, MNRAS, 288, 551 
Roberts, P. H. 1972, Phil. Trans. Roy. Soc. Lond., A224, 663 Roberts, P. H., \& Soward, A. M. 1975, Astron. Nachr., 296, 49 Rüdiger, G., \& Brandenburg, A. 1995, A\&A, 296, 557 Ruelle, D., \& Takens, F. 1972, Comm. Math. Phys., 20, 167 Schou, J., et al. 1998, ApJ, 505, 390

Schmitt, J. H. M. M., \& Rosner, R. 1983, ApJ, 265, 901

Spiegel, E. A., \& Weiss, N. O. 1980, Nature, 287, 616

Tavakol, R., \& Tworkowski, A. S. 1984, Phys. Lett. A, 130, 217

Tavakol, R., Tworkowski, A. S., Brandenburg, A., Moss, D., \& Tuominen, I. 1995, A\&A, 296, 269

Thompson, M. J., et al. 1996, Science, 272, 1300

Tobias, S. M. 1996, A\&A, 307, L21 (T96)

Tobias, S. M. 1997, A\&A, 322, 1007 (T97)
Tobias, S. M., Brummell, N. H., Clune, T. L., \& Toomre, J. 1998, ApJ, 502, L177

Tobias, S. M., Brummell, N. H., Clune, T. L., \& Toomre, J. 2001, ApJ, 549,1183

Tobias, S. M., Weiss, N. O., \& Kirk, V. 1995, MNRAS, 273, 1150

Torkelsson, U., \& Brandenburg, A. 1994a, A\&A, 283, 677

Torkelsson, U., \& Brandenburg, A. 1994b, A\&A, 292, 341

Tworkowski, A., Tavakol, R., Brandenburg, A., et al. 1998, MNRAS, 296, 287

Vainshtein, S. I., \& Cattaneo, F. 1992, ApJ, 393, 165

Vainshtein, S. I., \& Zel'dovich, Ya. B. 1972, Sov. Phys. Usp., 15, 159

Zel'dovich, Ya. B. 1956, Sov. Phys. JETP, 4, 460 